\title{
cDNA-AFLP analysis of gene expression differences between the flower bud and sprout-shoot apical meristem of Angelica sinensis (Oliv.) Diels
}

\author{
Guang $\mathrm{Yu}^{1}$, Jinao Duan ${ }^{1}$, Hui $\mathrm{Yan}^{1}$, Bingsheng Song ${ }^{2}$ and Ziqing $\mathrm{He}^{2}$ \\ ${ }^{1}$ Nanjing University of Chinese Medicine, Nanjing, China. \\ ${ }^{2}$ Gansu Mingui Chinese Medicinal Materials Technology Co., Ltd., Lanzhou, China.
}

\begin{abstract}
Angelica sinensis (Oliv.) Diels (Umbelliferae) is a well-known medicinal plant mainly distributed in Gansu Province of China. Its local and global demand is significant because of its food and medicinal applications. However, the early bolting rate of Angelica sinensis (Oliv.) Diels reaches 20\%-60\%, which seriously affects its food and medicinal qualities. Thus, differences in gene expression between the flower bud and sprout-shoot apical meristem underwent analysis, by means of cDNA-amplified restriction fragment length polymorphism, to better understand the flowering mechanism. 64 primer sets, each of which amplified to 60 transcript-derived fragments (TDFs), were used. Among these TDFs, 26 were expressed specifically in the flower bud. After cloning and sequencing, 32 distinct sequences were obtained from these 26 TDFs, and 25 were found with homologous sequences in databases. Confirmation of differential expression of 13 sequences was obtained by semi-quantitative RT-PCR, their showing higher expression levels in flower buds. These homologous sequences encode transposable elements, pentatricopeptide repeatcontaining proteins, DNA-binding transcription factors, zinc finger (B-box type) family proteins, NADP-dependent sorbitol 6-phosphate dehydrogenase (S6PDH), amongst others.
\end{abstract}

Key words: cDNA-AFLP, early bolting, Angelica sinensis (Oliv.) Diels, gene expression, TDFs.

Received: June 25, 2010; Accepted: February 7, 2011.

Angelica sinensis (Oliv.) Diels (Umbelliferae) is a world-famous medicinal plant distributed throughout Gansu Province, China (The State Pharmacopoeia Commission of P.R. China, 2005). The root of A. sinensis, called Danggui, is a popular tonic, having been recorded as early as A.D. 25-225 in the book "Shen Nong Ben Cao Jing" in the Han Dynasty. It is recommended as a tonic, hematopoietic, and anti-inflammatory agent in the treatment of menstrual disorders, amenorrhea, dysmenorrhea, etc. (Nanjing University of Chinese Medicine, 2006; Wang et al., 2007). It is also a health-food product for women in Asia (Yang et al., 2007), and a dietary supplement in Europe and America (Zhao et al., 2003; Deng et al., 2006). Thus, as a consequence of its wide variety of food and medicinal applications, demand is high.

However, in Gansu Province where it originated, the quality of Angelica sinensis has been seriously affected by its high early bolting rate. This results in low yield, thereby constituting a restriction to further development for numerous applications. Scientists have therefore begun to focus on the ecological factors involved, as well as the nutritional status of the plant itself (Lin et al., 2007; Wang et al., 2007)

Send correspondence to Jinao Duan. Nanjing University of Chinese Medicine, 138 Xianlin Road, Nanjing City, 210046 Jiangsu Province, China. E-mail: dja@njutcm.edu.cn. rather than on specific molecular mechanisms. Currently, no effective methods for the control and prevention of this characteristic exist, although the intense demand presses for an urgent solution.

From studies of Arabidopsis, rice, and other model plants, researchers have found that plant bolting is affected by gene expression and environmental factors. Four pathways affect flowering time, viz., the photoperiod, vernalization, autonomous and gibberellin pathways (Yanovsky et al., 2003; Komeda, 2004). In the present study, emphasis was placed on analyzing gene expression differences between the flower bud and sprout-shoot apical meristem, as well as on identifying certain key genes regulating bolting in Angelica sinensis.

cDNA amplified fragment length polymorphism (cDNA-AFLP) analysis with high reproducibility can be used to systematically screen a large number of differentially expressed cDNAs (Money et al., 1996; Habu et al., 1997; Milioni et al., 2002). This technique was employed hereby with 64 primer sets, to compare gene expression profiles in early-bolting and normal Angelica sinensis (Oliv) Diels. About 3000 transcript-derived fragments (TDFs) were amplified.

Angelica sinensis, a triennial medicinal plant, is characterized by early bolting. This is frequent in May. Thus, each year at this period, flower buds and sprout-shoot api- 
cal meristems were gathered from plants in Gansu Province. Flower buds of early-bolting plants, each around $0.5 \mathrm{~cm}$ in size, were harvested prior to full-blooming, together with sprout-shoot apical meristems from normal ones. The samples were frozen in liquid nitrogen and stored at $-80^{\circ} \mathrm{C}$. Total RNA was extracted from the plant material, by using the Trizol extraction method (Invitrogen, USA). RNA integrity and quantity were determined by running $2 \mu \mathrm{L}$ of total RNA in a formamide denaturing gel. First and second strand cDNA synthesis was according to manufacturer's instructions (Promega Universal Riboclone cDNA Synthesis System, USA). The resulting double-stranded cDNA was separated on agarose gel to check the sizes of cDNA samples.

cDNA-AFLP was carried out according to the procedure of Habu et al. (1997) with minor modifications. About $500 \mathrm{ng}$ of double-stranded cDNA underwent standard AFLP template production. The restriction enzymes used for cDNA digestion were $E c o R I 10 U / \mu L$, Sangon, China) and $M s e \mathrm{I} 10 \mathrm{U} / \mu \mathrm{L}$, Sangon, China). The digested products were ligated to adapters with the following sequences: EcoRI adapter - 5'-CTCGTAGACTGCGTACC-3' and 5'-AATTGGTACGCAGTC-3'; MseI adapter 5'-GACGATGAGTCCTGA G-3' and 5'-TACTCAGGAC TCAT-3'. The ligated products were preamplified with the corresponding preamplification primers (EcoRI: 5'-GACTGCGTACCAATTC -3', MseI: 5'-GA TGAGTCC TGAGTAA-3'). Twenty preamplification cycles were undertaken in a Bio-Rad MyCyler PCR system (Bio-Rad, USA). The PCR program was carried out as follows: $30 \mathrm{~s}$ at $94{ }^{\circ} \mathrm{C}, 30 \mathrm{~s}$ at $56^{\circ} \mathrm{C}$, and $1 \mathrm{~min}$ at $72{ }^{\circ} \mathrm{C}$. The final template was diluted 50 -fold with sterile water, in preparation for selective amplification.

Equal amounts of preamplified products were amplified with primers having selective nucleotides. The primers used for selective amplification were MseI+NN 5'-GATGAGTCCTGAGTAANN-3' and EcoRI+NN 5'-GACTGCGTA CCAATTCNN-3', in which N could correspond to either $\mathrm{A}, \mathrm{C}, \mathrm{G}$, or $\mathrm{T}$. The first cycle consisted of $30 \mathrm{~s}$ at $94^{\circ} \mathrm{C}, 30 \mathrm{~s}$ at $65^{\circ} \mathrm{C}$, and $1 \mathrm{~min}$ at $72^{\circ} \mathrm{C}$. For the next 13 cycles, the annealing temperature was reduced by $0.7^{\circ} \mathrm{C}$ per cycle. The final 23 cycles consisted of $30 \mathrm{~s}$ at $94^{\circ} \mathrm{C}, 30 \mathrm{~s}$ at $56^{\circ} \mathrm{C}$, and $1 \mathrm{~min}$ at $72^{\circ} \mathrm{C}$. Four sets of the EcoRI primer and 16 of the MseI primer were combined to give a total of 64 primer-pair combinations. For high-throughput analysis of differentially expressed TDFs, the PCR products were separated on denaturing $6 \%$ polyacrylamide gels.

The polymorphic TDFs based on presence, and expressed at higher levels in the flower buds, were cut from the gel. Maximum care was taken to avoid any contaminating fragment(s). They were initially eluted in $50 \mu \mathrm{L}$ of sterile double-distilled water at $95{ }^{\circ} \mathrm{C}$ for $15 \mathrm{~min}$ and then hydrated overnight at $4{ }^{\circ} \mathrm{C}$. Two $\mu \mathrm{L}$ of the aliquot was used for reamplification to a total volume of $20 \mu \mathrm{L}$, using the same set of corresponding selective primers and PCR conditions, as for selective amplification, except for an annealing temperature of $56^{\circ} \mathrm{C}, 35$ cycles, and a final $5 \mathrm{~min}$ extension. The PCR products were resolved in a $1.5 \%$ 1xTAE-agarose gel, each single-band being isolated and eluted with a Takara gel extraction kit (Takara, Japan). All PCR applications were carried out in the Bio-Rad MyCyler PCR system (Bio-Rad, USA). The eluted TDFs were cloned into a plasmid PMD-18T vector (Sangon, China), according to manufacturer's protocol. Five colonies were selected for sequencing analysis of each TDF. 130 colonies from 26 TDFs were selected and sequenced. The resultant sequences were analyzed for homologs by BLAST searches against public nonredundant databases (GenBank, Arabidopsis Information Resource, and Oryzabase) using BLASTN and BLASTX algorithms (Altschul et al.,1997).

Semi-quantitative reverse transcription-PCR (sqRTPCR) was carried out on $2 \mu \mathrm{g}$ of total RNA, by using a two-step RT-PCR kit (Takara, Japan), according to manufacturer's instructions. Primers were designed from the TDF sequences using a Primer 3.0 web resource. Total RNA was used as the internal RT-PCR standard for checking the quality of the RNA template. PCR conditions were essentially the same as those described earlier for reamplification of TDFs. In order to obtain reproducibility of results, sqRT-PCR was repeated three times.

64 primer combinations (Table S1) were used for AFLP. Only fragments over $80 \mathrm{bp}$ in size were selected for analysis. On an average, 60 clear and unambiguous bands (TDFs) were generated with each primer combination. Although most of these combinations produced identical patterns, 26 distinct TDFs were expressed at a higher level in the flower bud (Figure 1). This implied that minor changes in gene expression could account for the observed phenotypes of early bolting in Angelica sinensis.

These 26 TDF sof high expression level were excised from the polyacrylamide gels and re-amplified with the original primer sets used for cDNA-AFLP analysis. After the isolated TDFs were cloned, five colonies were selected and sequenced for each, thus giving 130 randomly selected colonies. Among these, 32 were distinct, this implying that each TDF corresponded to 1.23 distinct nucleotide sequences. A number of DNA sequences of the same length have been reported to co-migrate as a single AFLP fragment (Chen et al., 2008). As these migrate to the same position, AFLP resolution potential is low, in terms of variable base composition in DNA molecules of the same length. Thus, the 32 sequences were subjected to homology searches using BLAST against the databases, whereby 26 different sequences were obtained (Table 1), with six not resulting in any hits, this implying their possible correspondence to novel genes related to early bolting in Angelica sinensis (Oliv.) Diels.

Primer pairs were designed for 13 of the 32 distinct sequences obtained (Table 2). Differential expression of 


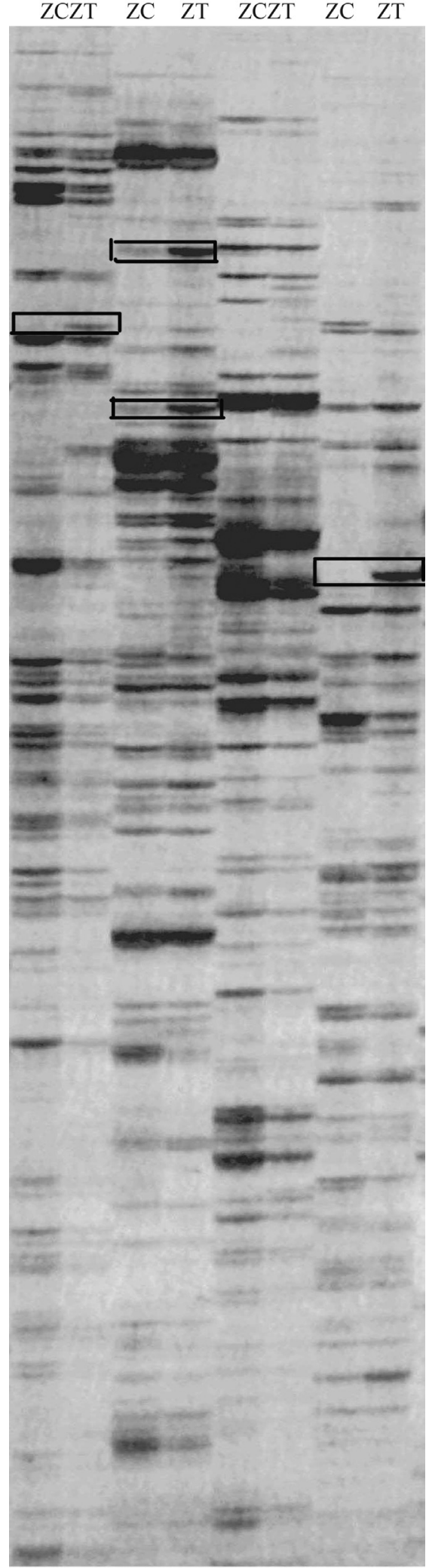

Figure 1 - cDNA-AFLP autoradiogram of four of the most informative-primer combinations that amplified differentially expressed genes in flower buds and sprout-shoot apical meristems. The codes for the primers used in the combinations are shown shown in Table S1, The boxes emphasize differential-expressed fragments. (ZT: flower bud, ZC: sprout-shoot apical meristem) the 13 distinct sequences was analyzed using sqRT-PCR, whereby it was shown that these sequences were expressed at a higher level in early bolting Angelica sinensis (Figure 2). TDFs showed different levels of homology with the genes/cDNAs of other species (with an E value ranging from 6.1 to $2 \mathrm{e}^{-17}$ ).

cDNA-AFLP, a variation of AFLP and derived from RNA fingerprint identification technology, has already become a sophisticated research tool for identifying differences in gene expression. This technique was here employed with 64 primer sets, to compare gene-expression profiles of flower buds and sprout-shoot apical meristems. We obtained 32 different sequences, some of which are possibly applicable to controlling early bolting. In this study, sequences with low E-value and definite functions were focused upon, thereby resulting in the identification of an RF2 protein (TDF A104-1), homeobox protein 25 (TDFA035-4), CMGC Ser/Thr protein kinase family (TDF A011-4), NADP-dependent sorbitol 6-phosphate dehydrogenase (TDF A021-3), ATAF-like NAC-domain transcription factor (TDF A010-1), ATTRX H1(TDF A110-1), and senescence-associated protein (TDF A115-1), all involved in cellular pathways leading to bolting.

TDF A104-1, as shown in Table 1, is matched with the RF2 protein. The $r f 2$ gene is one of the two nuclear genes required for fertility restoration in male-sterile T-cytoplasm (cmsT) plants. RF2 is an aldehyde dehydrogenase, thereby inferring several mechanisms that might explain Rf2-mediated fertility restoration in cmsT maize. Aldehyde dehydrogenase, possibly involved in the detoxification of acetaldehyde produced by ethanolic fermentation during pollen development, may also play a role in energy metabolism (Cui et al., 1996).

On the other hand, TDF A035-4 showed similarity with homeobox protein 25, which encodes ZFHD2, a member of the zinc-finger homeodomain transcription factor family. The cysteine-rich zinc-binding motifs are known as RING and B-box in several unrelated proteins. Structural, biochemical, and biological studies have revealed that these motifs may mediate protein-protein interactions (Borden, 1998).

TDF A011-4, which contains a protein kinase domain, belongs to the protein kinase superfamily (CMGC Ser/Thr protein kinase family, GSK-3 subfamily). It may mediate extracellular signals that regulate transcription in differentiating cells (Yamada, 2003). The expression patterns of SHAGGY-related protein kinase genes during wild-type Arabidopsis inflorescence development, detected by in situ hybridization, have been shown to be consistent with a possible role in floral meristem patterning. SHAGGY-related protein kinase gene transcripts were detected both at the periphery of the inflorescence meristem and within the floral meristem. At later stages, their expression became localized in specific regions of developing flower-organ primordia. The plants themselves developed 
flowers with a higher number of perianth organs and an alteration in the apical-basal patterning of the gynoecium (Dornelas, 2000).

TDF A021-3 is matched with the NADP-dependent sorbitol 6-phosphate dehydrogenase (S6PDH) gene. Sor- bitol-6-phosphate is a major photosynthetic product of several members of the Rosaceae family.

Transposons are mobile DNA molecules existing in the genomes of many organisms. The transposon superfamilies of higher plants were introduced as including LTR

Table 1 - Nucleotide homology of the transcript derived fragments (TDFs) with known gene sequences in non-redundant public databases. Shown are BLASTN results along with their respective similarity values.

\begin{tabular}{|c|c|c|c|c|c|c|c|c|}
\hline AFLP fragment & dbEST_Id & GenBank accession number & \multicolumn{5}{|l|}{ Similarity } & E-value \\
\hline A117-1 & 700623271 & HO056150 & \multicolumn{5}{|c|}{ retrotransposon protein, putative, Ty1-copia subclass } & $5 e-22$ \\
\hline A115-1 & 70062325 & HO056148 & \multicolumn{5}{|c|}{ senescence-associated protein } & 1.1 \\
\hline A112-1 & 70062323 & HO056146 & \multicolumn{5}{|c|}{ Reverse transcriptase (RNA-dependent DNA polymerase) } & $9.7 \mathrm{e}-06$ \\
\hline A110-1 & 70062322 & HO056145 & \multicolumn{5}{|c|}{ ATTRX H1, ATTRX1 } & 0.24 \\
\hline A107-1 & 70062320 & HO056143 & \multicolumn{5}{|c|}{ Ribosomal protein S8 (rps8) gene } & $3 e-54$ \\
\hline A104-1 & 70062319 & HO056142 & \multicolumn{5}{|l|}{$\mathrm{RF} 2$ protein } & $2 \mathrm{e}-44$ \\
\hline A103-2 & 70062318 & HO056141 & \multicolumn{5}{|c|}{$\begin{array}{l}\text { GLR2.6(member of Putative ligand-gated ion channel subunit } \\
\text { family) }\end{array}$} & 2.9 \\
\hline A102-3 & 70062317 & HO056140 & \multicolumn{5}{|c|}{$\begin{array}{l}\text { zinc finger (C3HC4-type RING finger) family protein / ankyrin } \\
\text { repeat family protein }\end{array}$} & 1e-02 \\
\hline A040 & 70062301 & HO056124 & \multicolumn{5}{|c|}{ transposable element gene; CACTA-like transposase family } & 0.54 \\
\hline A038-1 & 70062302 & HO056125 & \multicolumn{5}{|c|}{ pentatricopeptide (PPR) repeat-containing protein } & 26 \\
\hline A037-2 & 70062303 & HO056126 & \multicolumn{5}{|c|}{ Putative 22 kDa kafirin cluster; Ty3-Gypsy type } & $9 e-14$ \\
\hline A035-4 & 70062305 & HO056128 & \multicolumn{5}{|c|}{ Homeobox protein 25} & $1.7 \mathrm{e}-09$ \\
\hline A034-3 & 70062306 & HO056129 & \multicolumn{5}{|c|}{$\begin{array}{l}\text { zinc finger (B-box type) family protein; Identical to the Zinc finger } \\
\text { protein CONSTANS-LIKE } 13 \text { (COL13) }\end{array}$} & 6.1 \\
\hline $\begin{array}{l}\text { A033-4 } \\
\text { A116-1 } \\
\text { A109-2 }\end{array}$ & 70062307 & HO056130 & \multicolumn{5}{|c|}{$\begin{array}{l}\text { transposable element gene; } \\
\text { gypsy-like retrotransposon family }\end{array}$} & $5.5 \mathrm{e}-13$ \\
\hline A032-3 & 70062308 & HO056131 & \multicolumn{5}{|c|}{ retrotransposon protein } & $1 \mathrm{e}-13$ \\
\hline A021-3 & 70062309 & HO056132 & \multicolumn{5}{|c|}{$\begin{array}{l}\text { Prunus umbellata } \\
\text { NADP-dependent sorbitol } \\
\text { 6-phosphate dehydrogenase (s6pdh) gene }\end{array}$} & $2 \mathrm{e}-17$ \\
\hline A011-4 & 70062311 & HO056134 & \multicolumn{5}{|c|}{ serine /threonine kinase-like protein } & $1.2 \mathrm{e}-02$ \\
\hline A010-1 & 70062310 & HO056133 & \multicolumn{5}{|c|}{ ATAF-like NAC-domain transcription factor } & $6.4 \mathrm{e}-17$ \\
\hline A009-1 & 70062312 & HO056135 & \multicolumn{5}{|c|}{ putative reverse transcriptase } & $7.9 \mathrm{e}-09$ \\
\hline A008-4 & 70062314 & HO056137 & \multicolumn{5}{|c|}{$\begin{array}{l}\text { transposable element gene; non-LTR retrotransposon family } \\
\text { (LINE) }\end{array}$} & 2.5 \\
\hline A007-3 & 70062313 & HO056136 & \multicolumn{5}{|c|}{ transposable element gene, pseudogene, hypothetical protein } & 1.1 \\
\hline A006-4 & 70062315 & HO056138 & \multicolumn{5}{|c|}{ photosystem II protein Z [Daucus carota]protein } & $4 e-16$ \\
\hline A001-4 & 70062316 & HO056139 & \multicolumn{5}{|c|}{ transposable element gene; copia-like retrotransposon family } & $4.2 \mathrm{e}-09$ \\
\hline TDF-110-1 & TDF-A040 & TDF-A035-4 & TDF-A034-3 & TDF-A033-4 & TDF & $021-3$ & TDF & $-A 011-4$ \\
\hline $\mathrm{ZC}$ & $\mathrm{ZC}$ & $\mathrm{ZC}$ & $\mathrm{ZC}$ & $\mathrm{ZC}$ & $\mathrm{ZT}$ & $\mathrm{ZC}$ & $\mathrm{ZT}$ & $\mathrm{ZC}$ \\
\hline TDF-A010-1 & TDF-A009-1 & TDF-A008-4 & TDF-A007-3 & TDF-A006-4 & TDF- & $01-4$ & $\mathrm{Co}$ & introl \\
\hline ZT & $\mathrm{ZC}$ & $\mathrm{ZC}$ & $\mathrm{ZC}$ & $\mathrm{ZC}$ & $\mathrm{ZT}$ & $\mathrm{ZC}$ & ZT & $\mathrm{ZC}$ \\
\hline
\end{tabular}

Figure 2 - Confirmation, by semi-quantitative RT-PCR, of a higher level of TDF expression in the flower bud of early-bolting Angelica sinensis (Oliv.) Diels.(ZT: flower bud, ZC: sprout-shoot apical meristem). 
Table 2 - Sequences of the primers used for RT-PCR.

\begin{tabular}{lll}
\hline TDF clone & Forward prime (5-3) & Reverse primer (5-3) \\
\hline A040 & CAATCAGGCTCAACCTGTCA & GTGAAACAAGGGTGTCATCG \\
A035-4 & CCAGCATTTTGTGCCCTACT & CTGCTCCGCGGTAAACTTAG \\
A034-3 & TGGCTTAAAATCCTAGGGACAA & AAATCTGTTGGTTGGGTTTATG \\
A033-4 & TTCAGCTCTCGATAGTCTATGCAC & ACACAGTTGCAAGATCTTCTGG \\
A021-3 & GGCATGCAAAAACAGTCTGA & CATGAACTTGGCAAGCGTAA \\
A010-1 & GCGTACCAATTCGGAGAAAA & CCAACATATTTAGCCCCACTG \\
A011-4 & GCCCCAAAACAGCTTCAAAT & GCGTTTGTACTCAGGCTTTTG \\
A009-1 & CGAGGAAACCCCGTAGTTCT & AGTTGCATATTTGGGGCATC \\
A008-4 & CAACCGTTGATGAGTCAGGA & TTTTTCTCCCTTCGCTACAAA \\
A007-3 & CGTACCAATTCGGGGTTTT & CAACCGTTGATGAGTCAGGA \\
A006-4 & ACGCATCATGCAGTGCTAGT & GGGTCGTTTCAATTTTGGAT \\
A001-4 & ACTGCGTACCAATTCGGAAA & CCTGAGTAAGGTACGATTCTGGA \\
A110-1 & CATGGTGACCGTGCTCATTA & CTCAGACGTGGTCGAGTCAA \\
\hline
\end{tabular}

retrotransposons, hAT, CACTA elements, Mutator and MULEs, Tc1/mariner, miniature inverted repeat transposon MITEs, and so on. TDF A117-1, A112-1, A037-2, A033-4, A116-1, A109-2, A010-1 and A001-4 are matched with the transposable-element gene. TDF A010-1 and an ATAF-like NAC-domain transcription factor are homologous. Subtractive EST analysis, and the screening of cDNA libraries derived from Brassica napus leaves subjected to mechanical wounding, flea beetle feeding, or cold temperatures, revealed eight genes encoding NAC-domain transcription factors. These genes were found to be differentially regulated in response to biotic and abiotic stress, such as wounding, insect feeding, Sclerotinia sclerotiorum infection, cold shock and dehydration (Hegedus et al., 2003). Transposable elements may have an important effect on earlier bolting in Angelica sinensis.

TDF A110-1 is homologous to ATTRX H1. The latter encodes a cytosolic thioredoxin that reduces the disulfide bridges in target proteins, by the reversible formation of a disulfide bridge between the two neighboring Cys residues, present at the active site. Thioredoxins have been found to regulate a variety of biological reactions in prokaryotic and eukaryotic cells.

TDF A115-1 is homologous to senescence-associated genes (SAGs). A comparison was undertaken of the expression of several Arabidopsis thaliana SAGs in attached and/or detached leaves, as a possible response to age, dehydration, darkness, abscisic acid, cytokinin, and ethylene treatments. For the majority, the response to most of the treatments was similar. Detachment in darkness and ethylene were the strongest inducers of both SAGs and visible yellowing. Detachment in light, although a strong inducer of SAGs, was not of visible yellowing. The other treatments varied more in their individual effects. Responses, examined in both older and newer leaves, were generally much stronger in the former. As individual SAGs differed from the norms in various ways, this implies that their gene products play a role in overlapping, but not identical, circumstances. Some SAGs responded quickly to treatments, possibly indicating a direct response. Others responded more slowly, which may indicate an indirect response via treatment-induced senescence (Louis et al., 1998).

Early bolting in Angelica sinensis is related to general metabolism, transcription, signal transduction, and transposable elements (Table 1). It is a complex physiological action.

The remaining primer sets were used in analyzing the various genes expressed in early-bolting and normal Angelica sinensis plants. More of the TDFs involved in early bolting regulation need to be cloned, since some could be of possible use in preventing this phenomenon.

\section{Acknowledgments}

We are grateful to the National Natural Science Foundation of China (Project No.30801518), the National Science and Technology Pillar Program, Eleventh Five-Year Plan Period (Project No. 2006BA109B05-01; Project No. 2007BA137B02) and and A Project Funded by the Priority Academic Progam Development of Jiangsu Higher Education Institutions (PAPD) for the support they provided.

\section{References}

Altschul SF, Madden TL, Schaffer AA, Zhang J, Zhang Z, Miller W and Lipman DJ (1997) Gapped BLAST and PSI-BLAST: A new generation of protein database search programs. Nucleic Acids Res 25:3389-3402.

Bendahmane A, Budar F and Mireau H (2008) Characterization of Raphanus sativus pentatricopeptide repeat proteins encoded by the fertility restorer locus for Ogura cytoplasmic male sterility. Plant Cell 20:3331-3345. 
Borden KL (1998) RING fingers and B-boxes: Zinc-binding protein-protein interaction domains. Biochem Cell Biol 76:351-358.

Chen WH, Tseng IC, Tsai WC, Chiang MS, Chen YH and Chen $\mathrm{HH}$ (2006) AFLP fingerprinting and conversion to STS markers for identification of Oncidium cultivars. J Hortic Sci Biotechnol 81:791-796.

Cui X, Wise RP and Schnable PS (1996) The rf2 nuclear restorer gene of male-sterile T-cytoplasm maize. Science 272:13341336.

Deng SX, Chen SN, Yao P, Dejan N, Richard BB, Judy LB, Harry HSF, Norman RF and Guido FP (2006) Serotonergic activity-guided phytochemical investigation of the roots of $\mathrm{An}$ gelica sinensis (Oliv.) Diels. J Nat Prod 69:536-541.

Dornelas MC, Van Lammeren A and Kreis M (2000) Arabidopsis thaliana SHAGGY-related protein Kinases (AtSK11 and 12) function in perianth and gynoecium development. Plant J 21:419-429.

Habu Y, Fukada-Tanaka S, Hisatomi Y and Iida S (1997) Amplified restriction fragment length polymorphism-based mRNA fingerprinting using a single restriction enzyme that recognize a 4-bp sequence. Biochem Biophys Res 219:824828.

Hegedus D, Yu M, Baldwin D, Gruber M, Sharpe A, Parkin I, Whitwill S and Lydiate D (2003) Molecular characterization of Brassicanapus NAC domain transcriptional activators induced in response to biotic and abiotic stress. Plant Mol Biol 53:383-397.

Komeda Y (2004) Genetic regulation of time to flower in Arabidopsis thaliana. Annu Rev Plant Biol 55:521-535.

Lin MH and Qiu YD (2007) Effect of root diameter on early bolting rate and yield in seedling of Angelica sinensis (Oliv.) Diels. Chin Tradi Herbal Drugs 381386-1389.

Louis MW, Susheng G, Betania Q and Richard MA (1998) A comparison of the expression patterns of several senescence-associated genes in response to stress and hormone treatment. Plant Mol Biol 37:455-469.

Milioni D, Sado PE, Stacey NJ, Roberts K and Mcann MC (2002) Early gene expression associated with the commitment and differentiation of a plant tracheary element is revealed by cDNA-amplified fragment length polymorphism analysis. Plant Cell 14:2813-2824.

Money T, Reader S, Qu LJ, Dunford RP and Moore G (1996) AFLP-based mRNA fingerprinting. Nucleic Acids Res 24:2616-2627.

Mouradov A, Cremer F and Coupland G 2002 Control of flowering time: Interacting pathways as a basis for diversity. Plant Cell 14:111-130.
Nanjing University of Chinese Medicine (2006) Dictionary of Chinese Herbal Medicines. 2nd edition. Shanghai Science and Technology Press, Shanghai, pp 1207-1213.

The State Pharmacopoeia Commission of P.R. China (2005) The Pharmacopoeia of the People's Republic of China. Chemical Industry Publishing Press, Beijing, 89 pp.

Wang XZ, Lin HM and Liu XZ (2007) Effect of seedling size on the comprehensive agricultural characteristics and bolting rate of Angelica sinensis (Oliv.) Diels. J Gansu Agric Univ 10:59-63.

Wang YL, Liang YZ and Chen BM (2007) High-performance liquid chromatography with atmospjeric pressure chemical ionization and electrospray ionization mass spectromerry for analisis of Anegelica sinensis. Phytochem Anal 18:265-274.

Wu AY and Chen Y (2007) Study progress on Angelica sinens is early bolting. Gansu Agr Sci and Techn 3:20-23.

Yamada K, Lim J, Dale JM, Chen H, Shinn P, Palm CJ and Southwick AM (2003) Empirical analysis of transcriptional activity in the Arabidopsis genome. Science 302:842-846.

Yang XB, Zhao Y and Lv Y (2008) In vivo macrophage activation and physicochemical property of the different polysaccharide fractions purified from Angelica sinensis (Oliv.) Diels. Carbohydr Polym 71:372-379.

Yanovsky MJ and Kay SA (2003) Living by the calendar: How plants know when to flower. Nat Rev Mol Cell Biol 4:265276.

Zhao KJ, Dong TX, Tu PF, Song ZH, Lo CK and Tsim KWK (2003) Molecular Genetic and Chemical Assessment of Radix Angelica (Danggui) in China. J Agric Food Chem 51:2576-2583.

\section{Internet Resources}

GenBank, http://www.ncbi.nlm.nih.gov/BLAST.

The Arabidopsis Information Resource http://www. arabidopsis.org/cgi-bin/fasta/nph-TAIRfasta.pl.

Oryzabase, Integrated Rice Science Database, http://www.shigen.nig.ac.jp/rice/oryzabase/blast. Primer 3 software, http://frodo.wi.mit.edu/.

\section{Supplementary Material}

The following online material is available for this article:

Table S1 - Sequences of the primers used for AFLP.

This material is available as part of the online article from http://www.scielo.br/gmb.

\section{Associate Editor: Dario Grattapaglia}

License information: This is an open-access article distributed under the terms of the Creative Commons Attribution License, which permits unrestricted use, distribution, and reproduction in any medium, provided the original work is properly cited. 
Table S1 - Sequences of the primers used for AFLP.

\begin{tabular}{ll}
\hline MseI+NN & EcoRI+NN \\
\hline GATGAGTCCTGAGTAAAA & GACTGCGTA CCAATTCGA \\
GATGAGTCCTGAGTAAAT & GACTGCGTA CCAATTCGT \\
GATGAGTCCTGAGTAAAC & GACTGCGTA CCAATTCGC \\
GATGAGTCCTGAGTAAAG & GACTGCGTA CCAATTCGG \\
GATGAGTCCTGAGTAATA & \\
GATGAGTCCTGAGTAATC & \\
GATGAGTCCTGAGTAATG & \\
GATGAGTCCTGAGTAATT & \\
GATGAGTCCTGAGTAACA & \\
GATGAGTCCTGAGTAACT & \\
GATGAGTCCTGAGTAACC & \\
GATGAGTCCTGAGTAACG & \\
GATGAGTCCTGAGTAAGA & \\
GATGAGTCCTGAGTAAGT & \\
GATGAGTCCTGAGTAAGC & \\
GATGAGTCCTGAGTAAGG
\end{tabular}

